\title{
Innate Immune Tolerance and the Role of Kupffer Cells in Differential Responses to Interferon Therapy Among Patients With HCV Genotype 1 Infection
}

\author{
DARYL T.-Y. LAU ${ }^{1}$, AMINA NEGASH ${ }^{2}$, JIE CHEN ${ }^{1}$, NANETTE CROCHET ${ }^{2}$, MALA SINHA ${ }^{3,4}$, \\ YUHONG ZHANG ${ }^{1}$, JEREMIE GUEDJ ${ }^{5}$, SHARON HOLDER ${ }^{1}$, TAKESHI SAITO ${ }^{2}$, STANLEY $M$. \\ LEMON $^{6}$, BRUCE A. LUXON ${ }^{3,4}$, ALAN S. PERELSON ${ }^{5}$, and MICHAEL GALE Jr ${ }^{2}$ \\ ${ }^{1}$ Liver Center, Division of Gastroenterology, Department of Medicine, Beth Israel Deaconess \\ Medical Center, Harvard Medical School, Boston, Massachusetts \\ ${ }^{2}$ Center for the Study of Hepatitis C Virus Infection and Immunity, Department of Immunology, \\ University of Washington, Seattle, Washington \\ ${ }^{3}$ Biochemistry and Molecular Biology, University of Texas Medical Branch at Galveston, \\ Galveston, Texas \\ ${ }^{4}$ Institute of Translational Science, University of Texas Medical Branch at Galveston, Galveston, \\ Texas \\ ${ }^{5}$ Theoretical Biology \& Biophysics, Los Alamos National Laboratory, Los Alamos, New Mexico \\ ${ }^{6}$ Division of Infectious Diseases, Department of Medicine, University of North Carolina at Chapel \\ Hill, Chapel Hill, North Carolina
}

\begin{abstract}
BACKGROUND \& AIMS-In patients with hepatitis C virus (HCV) infection, interferon alfa (IFN- $\alpha$ ) alters expression of IFN-stimulated genes (ISGs), but little is understood about factors that determine outcomes of therapy. We used a systems biology approach to evaluate the acute response of patients with chronic hepatitis $\mathrm{C}$ to IFN- $\alpha$ therapy.
\end{abstract}

METHODS-We collected liver biopsy samples from 8 treatment-naïve patients with chronic HCV genotype 1 infection at baseline and 24 hours after treatment with IFN- $\alpha$-2a (10 MU subcutaneously). Blood samples were collected before and up to 48 hours after administration of IFN- $\alpha$-2a to measure HCV RNA levels and for gene expression analysis. Patients then received pegylated IFN- $\alpha-2 a$ and ribavirin on day 5 of the study; therapy continued for up to 48 weeks.

RESULTS-Based on the kinetics of HCV RNA during the first 12 weeks of therapy, 2 patients were rapid virologic responders, 4 were early virologic responders, and 2 did not respond to therapy (nonresponders). Nonresponders had high pretreatment levels of ISG expression in the liver but not in peripheral blood mononuclear cells. In responders, after administration of IFN- $\alpha$,

(C) 2013 by the AGA Institute

Address requests for reprints to: Daryl T.-Y. Lau, MD, MSc, MPH, Liver Center, Division of Gastroenterology, Department of Medicine, Beth Israel Deaconess Medical Center, Harvard Medical School, 110 Francis Street, Suite 4A, Boston, Massachusetts 02215.dlau@bidmc.harvard.edu; fax: (617) 632-1093.

Conflicts of interest

The authors disclose no conflicts.

Supplementary Material

Note: To access the supplementary material accompanying this article, visit the online version of Gastroenterology www.gastrojournal.org, and at http://dx.10.1053/j.gastro.2012.10.044. 
intrahepatic ISG expression increased significantly from baseline and was associated with a rapid phase 1 decrease in HCV. We identified distinct hepatic expression and tissue distribution patterns of ISGs that segregated with treatment outcome. Importantly, Kupffer cells were a local source of IFN that promoted basal expression of ISG in hepatocytes of non-responders. This finding was validated in cultured THP1 human macrophages that expressed IFN- $\beta$ after exposure to viable HCV 2a. When Huh7 K2040 and Huh7 L2198S hepatoma cells were incubated with IFN- $\alpha$-2a, expression of ISGs peaked by 4 hours and decreased by 72 hours, associated with an increase in level of HCV RNA. This indicates that constitutive exposure to IFN causes hepatoma cells to become tolerant of ISG function.

CONCLUSIONS-In patients with chronic HCV infection, IFN production by Kupffer cells might promote innate immune tolerance, characterized by a lack of response to IFN therapy. Strategies to disrupt the virus-host interactions that induce innate immune tolerance should improve therapy.

\section{Keywords}

PBMC; Microarray Analysis; Therapeutic Efficacy; Drug

Patients with hepatitis C virus (HCV) infection are treated with interferon (IFN)- $\alpha$-based therapy. ${ }^{1}$ Although the recent approval of the HCV-specific protease inhibitors marks a significant advance in the treatment of patients with chronic hepatitis $\mathrm{C}$, the combination of IFN- $\alpha$ and ribivirin remains the platform with which such direct-acting antivirals are used in HCV treatment. ${ }^{1}$ This underscores the need to understand the actions of IFN- $\alpha$ in vivo to design the most effective antiviral regimen for the treatment of patients with $\mathrm{HCV}$.

Treatment with IFN induces the expression of hundreds of interferon-stimulated genes (ISGs) in responding cells. ${ }^{2,3}$ ISG products serve as immune effectors to impart cellular response pathways of innate immunity and others to drive the global antiviral actions that suppress HCV infection. ${ }^{3}$ Moreover, IFN serves to support and modulate the adaptive immune response through specific ISGs expressed in immune cells. ${ }^{4,5}$ However, the systemwide and hepatic responses to IFN among patients with HCV and how these responses contribute to differential therapeutic outcomes are still not well understood. ${ }^{2}$

We specifically evaluated the acute response to IFN- $\alpha$ in vivo after the first 24 hours of treatment in patients with $\mathrm{HCV}$ genotype 1 infection and modeled this response in vitro. Our results show tissue- and cell-specific response compartmentalization that links local IFN- $\beta$ production by Kupffer cells to a hepatic ISG expression set point as a central feature of "innate immune tolerance" that mitigates the response to IFN therapy.

\section{Patients and Methods}

Detailed information on methods is available in Supplementary Patients and Methods.

Treatment-naïve white and black patients with HCV genotype 1 who were 18 years of age or older were enrolled in the study. Subjects underwent a baseline percutaneous liver biopsy on day 1 of the study. A single dose of IFN- $\boldsymbol{a}$ (10 MU subcutaneously) was administered on day 3 , and a repeat liver biopsy for research purposes was performed 24 hours after treatment. A total of 1 to $3 \mathrm{~cm}$ of the excess tissue was immediately preserved in a commercial solution (RNAlater; Ambion, Austin, TX) for Affymetrix analysis (Affymetrix, Inc, Santa Clara, CA). Fifteen-milliliter blood samples were drawn immediately before and $1,3,6,12,18,24,36$, and 48 hours after treatment with IFN for quantitation of HCV RNA and Affymetrix analysis. The first dose of pegylated IFN- $\alpha$-2a and weight-based ribavirin was administered on day 5 , and therapy continued for up to 48 weeks as per the standard of 
care recommendation. The clinical study was approved by the institutional review board at the University of Texas Medical Branch and Beth Israel Deaconess Medical Center. The first 2 subjects were enrolled at the University of Texas Medical Branch at Galveston, and all subsequent subjects were enrolled at Beth Israel Deaconess Medical Center. Study procedures were identical.

\section{HCV Kinetic Mathematical Modeling}

To analyze the early HCV RNA kinetics in patients, we fit via nonlinear least squares regression the natural logarithm of $V(t)$ predicted by the equation $V(t)=V_{d}(1-\epsilon+\epsilon$ $\left.\exp \left(-c\left(t-t_{0}\right)\right)\right)$, derived by Neumann et al to the natural logarithm of the serum viral load data. ${ }^{6}$ When the drug effectiveness $(\epsilon)=1$, the virion production is fully suppressed; if $\epsilon=0$, the production is unchanged. This equation is based on the assumption that the HCV RNA level was in steady state before the initiation therapy and that the infected cell number remained constant during the first 1 to 2 days of therapy. 6,7

\section{Liver and Peripheral Blood Mononuclear Cell Sample Preparation and Microarray Analysis}

Immediately after liver biopsy, a portion of the tissue was placed into RNAlater (Ambion) for storage at $-70^{\circ} \mathrm{C}$. Total RNA was extracted using the ToTALLY RNA Kit (Ambion). Affymetrix HG-U133 2.0 Human GeneChips were used for all microarray experiments. Microarray analyses were conducted exactly as described previously. ${ }^{8}$ The Affymetrix methods used for the peripheral blood lymphocyte samples are similar.

\section{Computational Analysis of Gene Expression Data Sets}

Pre-IFN-treated liver samples were analyzed using one-way analysis of variance and hierarchical clustering methods. The resulting genes that significantly differentiated the 3 patient groups at a $P$ value of $\leq 05$ were expressed in a heat map using Spotfire Decision Site software (TIBCO Spotfire, Somerville, MA). Functional and canonical pathways analysis was performed using Ingenuity Pathways Analysis (Ingenuity Systems, Redwood City, CA) on those significant genes with an absolute fold change of $\geq 1$.4. Paired $t$ test was performed on pre-IFN and post-IFN gene expression levels using the robust

MultiExperiment Viewer (MeV 4.0; Dana Farber Cancer Institute, Boston, MA). After hierarchical clustering, differentially expressed genes at a $P$ value of $\leq 001$ were expressed in a heat map using Spotfire Decision Site software. Genes with absolute fold change of $\geq 2$ were further analyzed by Venn analysis of the 3 groups. Detailed analysis of the peripheral blood mononuclear cell (PBMC) data set appears in Supplementary Patients and Methods.

\section{Cell Culture}

Huh7 and Huh7-K2040 are parental human hepatoma cells and a matching clonal cell line harboring the HCV 1b K2040 or L2198S subgenomic replicon, respectively, and were cultured as described. ${ }^{5}$ THP1 cells were maintained in culture medium supplemented with $10 \%$ fetal bovine serum, L-glutamine, and standard antibiotics and were differentiated into adherent macrophage-like cells by treatment with phorbol myristate acetate. Cells were treated, harvested, and analyzed as described in Supplementary Patients and Methods.

HCV preparation- $\mathrm{HCV}$ (strain JFH1) was produced in Huh7.5 cells exactly as described. ${ }^{9} \mathrm{HCV}$ was purified from cell culture supernatants through centrifugation over sucrose gradients. Control virus-free Huh7 cell culture medium was centrifuged through sucrose gradients for collection as a treatment-negative control. HCV infectivity was measured as described. ${ }^{9}$ Virion and media control tested negative for endotoxin as assayed using the limulus amebocyte lysate endotoxin assay (Cape Cod, Inc, Falmouth, MA). 


\section{Immunohistochemical Staining and Confocal Microscopy}

For analysis of sections of paraffin-embedded liver biopsies, we used a previously reported procedure that involved 4 major steps, including deparaffinization of tissue, antigen retrieval, tissue permeabilization, and immunostaining. ${ }^{8}$ Detailed procedures are described in Supplementary Patients and Methods.

\section{Results}

\section{Phase 1 Viral Kinetics Reveals Distinct Clinical Response Profiles to IFN Therapy}

Acute IFN- $a$ response profiling included 8 subjects (6 men and 2 women) chronically infected with $\mathrm{HCV}$ genotype 1. Pretreatment HCV RNA titers ranged from 1.5 to $13.6 \mathrm{MIU} /$ $\mathrm{mL}$ (Supplementary Table 1). Based on their HCV RNA kinetics during the first 12 weeks on therapy, 2 patients were classified as rapid virologic responders (RVRs), 4 were early virologic responders (EVRs), and 2 were nonresponders (NRs) (Supplementary Table 1). The mathematical model of Neumann et $\mathrm{al}^{6}$ was fit to the serial HCV RNA loads from each individual patient obtained during the first 24 hours (phase 1 kinetic) after a single 10 million unit dose of standard IFN- $\alpha$-2a. The 2 RVRs had a reduction in HCV RNA levels $\geq 2$ $\log _{10} \mathrm{IU} / \mathrm{mL}$ within 24 hours, and their drug effectiveness $(\epsilon)$ in suppressing virus production was $>0.995$ (Figure 1). In contrast, the 2 NRs had a decline in HCV RNA levels $\checkmark \log _{10} \mathrm{IU} / \mathrm{mL}$ within the same time interval and the $\epsilon$ was low (0.464 and 0.852 , respectively). The 4 EVRs had intermediate declines in HCV RNA levels, falling between the RVRs and NRs, and had a mean $\epsilon$ of 0.932 (Figure 1A).

\section{Hepatic Gene Expression Before Treatment and During the Phase 1 Response to IFN Differentiates Response Groups}

We analyzed global gene expression in pretreatment tissues from our cohort. Direct comparison of gene expression profiles among patients revealed 930 genes that significantly differentiate the 3 treatment responder groups at a $P$ value of $\leq 05$. As shown in Figure $1 B$, we observed a clear demarcation of the RVRs, EVRs, and NRs in 3 distinct gene clusters. Of these 930 genes, 635 (68\%) were increased in expression (up-regulated) in NRs compared with RVRs and EVRs. In contrast, there were only 143 and 123 genes (15\% and 13\%), respectively, that were up-regulated in RVRs and EVRs compared with NRs.

Computational analysis to assign response pathways to the 635 genes that were up-regulated among NRs compared with RVRs and EVRs was subsequently performed. A total of 265 of these genes belong to the categories of apoptosis $(36 \%)$, cell cycle (18\%), cell signaling (15\%), cellular development (14\%), lipid metabolism (9\%), and immune response (6\%). In total, these response pathways are indicative of the inflammatory and innate immune response to HCV infection. Among these genes, 41 were known ISGs and pathway modeling analysis identified STAT1, the major component of the IFN-induced transcription factor ISFG3,${ }^{10}$ as a central regulatory node of gene expression linked to a variety of ISGs (Figure $1 C$ and $D$ ). These results are consistent with IFN- $\alpha / \beta$ receptor signaling as the intracellular effector pathway driving the high basal ISG expression in pretreatment NRs compared with RVRs and EVRs (Figure $1 C$ and $D$ ). However, the activation of the IFN- $\lambda$ signaling pathway could be an alternative explanation based on the bioinformatics analysis. ${ }^{11}$

To assess the acute hepatic transcriptional response to treatment with IFN- $\alpha$, we examined gene expression in liver tissue recovered 24 hours after administration of IFN- $\alpha$-2a. Figure $2 A$ shows a hierarchical clustering of the 86 differentially expressed genes $(P \leq .001)$. These data show that the EVRs and RVRs together formed distinct gene clusters from NRs and feature a significant number of up-regulated genes 24 hours after administration of IFN- $a$. 
For the NRs, their pre-IFN and 24-hour post-IFN samples formed the same gene clusters, revealing an overall lack of further increase in gene expression during the acute response to IFN. Although the EVRs and RVRs had a higher number of genes expressed in response to IFN treatment, there was a conserved set of 28 genes that were reduced in expression or "down-regulated" in response to IFN (Supplementary Table 2).

Among the ISGs induced by treatment with IFN- $a$, there were members of the IFIT gene family. ISG56, the product of IFIT1, is a potent antiviral protein that restricts HCV RNA translation. ${ }^{12}$ Although expression of the IFIT1 genes can be induced through the HCVresponsive RIG-I pathway to activate IRF-3, IFIT1 expression is also highly induced by IFN. ${ }^{12}$ Because HCV can block the RIG-I pathway in infected cells, ${ }^{3,9}$ accordingly the basal ISG expression was low or not present in these patients (Figure $2 B$ ). Thus, the induction of ISGs is largely attributed to IFN signaling (Figure 2C). Additionally, a subset of ISGs with known antiviral potency, including oligoadenylate synthetase (OAS), interferon-induced transmembrane 1 (IFITM1), and the guanosine triphosphatase MX1, with expression induced through IFN- $\alpha / \beta$ receptor signaling, were identified. ${ }^{10}$ These ISGs were induced from a low or nonexpressed pretreatment level to a multiple-fold increase in RVRs and EVRs (Figure $2 B$ and $C$ ). By comparison, NRs exhibited a high pretreatment "set point" expression level of ISGs without further induction on treatment with IFN (Figure 2C). Venn analysis was performed to examine the induced genes and response pathways in RVRs and EVRs. Using criteria with a $P$ value of $\leq 05$ and fold changes $\geq 1.5,16$ unique ISGs common to RVRs and EVRs were identified in the Ingenuity Pathways Analysis (Supplementary Table 3). Pathway modeling assigned IRF-7, a STAT1-responsive gene and IFN-induced transcription factor that serves to amplify the IFN response, ${ }^{3,10}$ as a major node linking the expression of ISGs after IFN- $\boldsymbol{\alpha}$ among responders, suggesting that the rapid response of EVRs and RVRs to IFN involved response amplification through IRF-7 signaling (Figure $2 D$ and $E$ ).

\section{ISG Expression Patterns of the Acute Response to IFN Therapy}

To define the acute systemic response to IFN- $\alpha$, we conducted parallel comparison of the IFN-induced gene profiles between PBMCs and liver samples from the patient cohorts before and after treatment with IFN- $a$. Unlike the pretreatment hepatic gene profiles, the baseline ISG expression in PBMCs was generally silent and similar among RVRs, EVRs, and NRs (Supplementary Figure $1 A$ ). In all patients, induction of ISG was maximal in PBMCs within the first 3 to 12 hours but was overall lowest in the NRs (Supplementary Figure $1 B$ ). Thus, the pretreatment ISG expression set point and acute phase 1 response to IFN are distinct between liver and systemic peripheral blood compartments, as previously noted. ${ }^{13}$

We assessed both the tissue distribution and the cell type response to treatment with IFN. Biopsy tissue sections were immunostained with antibodies against specific ISG-encoded proteins and were analyzed by high-resolution laser scanning confocal microscopy. ${ }^{8}$ Tissues were costained in sets to detect nucleus and IFIT1/OAS1, IFITM1/MX1, and MX1 with CD68, a marker of Kupffer cells. ${ }^{14}$ In general, ISG expression was low or silent in pretreatment tissue from RVRs and EVRs but was induced to a highly abundant state 24 hours after treatment with IFN (Figure $3 A$ and $B$, upper and middle panels). Notably, OAS1 was expressed at a low basal level in all specimens examined, regardless of response outcome. In general, the hepatic ISGs were expressed to already high levels within pretreatment specimens from NRs (Figure $3 A-D$ ).

We further assessed IFN response patterns of ISG expression within the liver of treated patients and observed 3 distinct patterns of acute response to IFN. The first was observed only in tissue from EVRs and RVRs and was termed "confluent and overlapping" in that this 
response pattern occurred throughout the majority of the tissue section in which both ISG products of each staining set were induced and abundant across hepatocytes (Figure 3E, top panels). The second pattern was a "cell specific" response and occurred only within NRs wherein side-by-side cells expressed distinct levels of ISG product indicative of different IFN response potentials among each (Figure $3 F$, middle panels). The third response pattern was also observed in NR patient tissue as a "focal response" wherein IFN-induced protein expression was found only in focal regions of the tissue (Figure $3 G$, bottom panels). These latter response patterns were present in both pre-treatment and treatment NR tissue. Thus, the hepatic response to IFN exhibits distinctions in ISG expression pretreatment set point, uniformity, and cell type specificity that may impart a differential outcome to therapy for $\mathrm{HCV}$ infection. We found that in some cases the ISG expression pattern included specific differences in IFN-induced protein levels between hepatocytes and Kupffer cells, resident hepatic macrophages. In particular, we found that NR patient tissue often exhibited pretreatment Kupffer cell expression of MX1 surrounded by a halo of MX1-expressing hepatocytes (Figure 3F).

The basal ISG expression within hepatocytes and Kupffer cells observed within NRs suggests that IFN was being produced and secreted by Kupffer cells to drive a paracrine pretreatment IFN response. To assess whether Kupffer cells within the liver were producing IFN endogenously, we stained liver tissue with antibody specific for IFN- $\beta$ in combination with anti-CD68. As shown in Figure $4 A$, these analyses identified CD68 ${ }^{+}$Kupffer cells harboring low but detectable levels of IFN- $\beta$ specifically within NRs and showing a peripheral staining pattern indicative of ongoing IFN- $\beta$ secretion.

To determine if HCV exposure and/or uptake by Kupffer cells might induce their production of IFN, we modeled HCV exposure in vitro at a multiplicity of infection corresponding to 0.1 focus-forming units based on Huh7 cells per milliliter using differentiated THP1 human macrophage cells. Exposure of THP1 cells to viable HCV 2a but not control Huh7 culture media resulted in IFN- $\beta$ protein expression (Figure $4 B$ ). Moreover, exposure of THP1 to HCV $2 \mathrm{a}$ also induced low-level expression of IFN- $\beta$ messenger RNA (mRNA) (Figure $4 C$ ) in which IFN- $\beta$ was secreted into the culture supernatant of the former at $10 \mathrm{pg} / \mathrm{mL}$ as determined by specific enzyme-linked immunosorbent assay. By comparison, 24 hours of productive Sendai virus infection of THP1 cells induced a high level of IFN- $\beta$ mRNA (Figure $4 C$, far right bar). We found that HCV could actually enter THP1 cells and transiently produce viral proteins through a process that was insensitive to anti-CD81 blocking antibodies (Figure 4D, left panel). Viral proteins, including the HCV NS3 protease, were routinely detected in THP1 cells within 1 hour of HCV exposure, but their production was only transient such that they became undetectable at time points beyond 6 hours after virus exposure of cells (Figure 4D, right panel). Moreover, we observed MAVS expression in HCV-treated THP1 cells with no indication of MAVS cleavage by the viral NS3 protease, which targets and cleaves MAVS in infected hepatocytes as an immune evasion strategy. ${ }^{9}$ Lack of MAVS cleavage could be attributed to only low, transient levels of NS3 present in THP1 cells. We also observed increased RIG-I levels beginning 6 hours after HCV exposure of cells, consistent with the response to IFN- $\beta$. HCV RNA was present within THP1 cells after $\mathrm{HCV}$ exposure but decayed over 72 hours (Figure $4 E$, left panel), whereas viral RNA was amplified in Huh7 cells similarly exposed to HCV over this time course (Figure $4 E$, right panel). When introduced in THP1 cells via lipophilic transfection, HCV RNA itself was sufficient to trigger IFN- $\beta$ expression (data not shown). We therefore assessed IFN- $\beta$ mRNA expression in THP1 cells deficient in RLR or specific TLR signaling function through short hairpin RNA (shRNA) knockdown of MAVS or MyD88, respectively. THP1 cells harboring control nontargeting shRNA lentivirus or lentivirus-expressing shRNA against MAVS or MyD88 were transfected with the HCV poly-U/UC motif RNA, a potent pathogen-associated molecular pattern (PAMP) that stimulates IFN production (reviewed in 
Gale and Foy $^{3}$ ). PAMP RNA was sufficient to trigger IFN- $\beta$ expression in THP1 cells expressing nontargeting control shRNA or MyD88 shRNA, but IFN- $\beta$ expression was blocked in cells expressing MAVS shRNA (Figure $4 F$, left panel). Treatment of cells with cytochalasin $\mathrm{D}$, which prevents phagocytosis, significantly reduced the uptake of $\mathrm{HCV}$ in THP1 cells (Figure $4 F$, right panel). Treatment of cells with bafilomycin to disrupt endosome acidification and receptor-mediated endocytosis had no effect on HCV uptake (data not shown). These observations reveal phagocytosis as a critical cellular process in mediating HCV uptake by macrophages. Our results show that while HCV fails to replicate after macrophage uptake, the residual viral RNA can trigger intracellular MAVS-dependent signaling of IFN- $\beta$ expression. Thus, liver-resident myeloid lineage cells such as Kupffer cells may take up $\mathrm{HCV}$ through phagocytic actions to trigger IFN- $\beta$ production through endogenous RLR signaling processes of IFN expression.

To determine how constitutive ISG expression could affect the cellular response of IFN, we modeled the acute response to IFN therapy during chronic HCV RNA replication. Parallel cultures of Huh7 K2040 and Huh7 L2198S cells ${ }^{15,16}$ were treated with IFN. ISG and HCV

RNA levels were evaluated over a time course. Cultures were then repeatedly treated 3 times with IFN for 4 hours, and ISG expression and HCV RNA levels were measured after each treatment. At baseline before administration of IFN- $\alpha$-2a, ISG expression was minimal (Figure 5 $A$ and $B$ ). The expression of the ISGs in Huh7 K2040 and Huh7 L2198S cells peaked at 4 hours after treatment. By 72 hours, the levels of all ISGs were reduced. With repeated IFN- $\alpha$-2a treatments, there were progressively blunted responses of all ISGs examined. Importantly, after the final round of sustained IFN- $a 2$ a treatment, ISG levels exhibited a constitutively higher set point than their starting baseline values (see Figure $5 A$ ). However, by 72 hours, the HCV RNA level began to increase. IFN- $\alpha$-2a treatment of cells beyond the first 72-hour period failed to suppress HCV RNA levels and overall mimicked the NR patient phenotype of maintained viral load with an induced ISG set point. Because the decay in HCV RNA within Huh K2040 cells showed faster than expected kinetics, we also evaluated HCV RNA levels in IFN-treated Huh7 L2198S cells (Figure 5B). In this case, the HCV RNA exhibited relatively lower levels and slower decay of viral RNA but showed recovery similar to that of Huh7 K2040 cells to near-pretreatment levels after 72 hours of treatment with IFN. In control experiments, we replaced the culture media of the replicon cell lines with fresh media lacking IFN, and cultures were similarly assessed for HCV and ISG RNA expression. In the absence of further treatment with IFN, the ISG set point and HCV RNA levels returned to near baseline, implying that treatment with IFN imparts control of ISG and HCV RNA levels in this culture model (Supplementary Figure 3). The observed differences in HCV RNA decay rates between replicons reflect distinctions in adaptive mutations of each $\mathrm{HCV} \mathrm{RNA}^{15}$ and may also reflect clonal variation in RNA metabolism processes among cell lines. Flow cytometric analysis revealed that IFN- $\alpha / \beta$ receptor levels were increased on treatment with IFN- $\alpha$-2a and maintained at a high level throughout the duration of the experiment (Supplementary Figure 4). Thus, constitutive IFN exposure and high ISG set are linked with the NR phenotype in vitro. These results imply that constitutive exposure to IFN and actions of ISGs may drive a state of tolerance against the therapeutic actions of IFN- $\alpha$ in which the hepatocyte is unable to fully respond and suppress HCV.

\section{Discussion}

$\mathrm{HCV}$ can induce producton of IFN during de novo infection of hepatocytes as the virus spreads from cell to cell within cell culture models and in the liver in chronic HCV. ${ }^{8,9}$ Secreted IFN- $\beta$ induces a tissue-wide antiviral state via paracrine signaling to induce ISG expression. ${ }^{3}$ However, within infected hepatocytes, the viral NS3/4A protein targets and cleaves MAVS to suppress RIG-I signaling and ablate IFN production by hepatocytes. ${ }^{9,17}$ 
Our results now define Kupffer cells as a possible source of hepatic IFN and suggest that local IFN- $\beta$ production by Kuppfer cells may drive ISG set point levels in surrounding hepatocytes. Similarly, plasmacytoid dendritic cells can produce IFN when exposed to $\mathrm{HCV},{ }^{18}$ and we have shown that plasmacytoid dendritic cells are abundantly present in the liver in chronic $\mathrm{HCV}^{8}$ to possibly contribute a source of IFN. ${ }^{18}$ Although our study has a limited sample size, the observations that hepatic ISG set point levels were inversely associated with the outcome of IFN therapy are consistent with other studies. ${ }^{13,19-21}$ Thus, hepatic IFN production from myeloid cell sources may underlie the poor outcome to IFN therapy in these patients. Chen et al observed higher ISG15 levels within Kupffer cells of responders. However, ISG15 expression was higher within hepatocytes of NRs. ${ }^{22}$ Expression and function of various, specific ISGs may thereby impart a differential therapy outcome through cell-specific antiviral actions.

We show that Kupffer cells express IFN- $\beta$ protein in vivo and that $\mathrm{HCV}$ can simulate macrophage cell model induction of IFN on cell exposure in vitro. Our results reveal that HCV can enter THP1 cells through phagocytic uptake, showing that HCV does not replicate but instead decays after cell uptake. Because HCV RNA alone was sufficient to stimulate THP1 cell IFN production, and IFN induction was blocked by knockdown of MAVS but not MyD88 expression, we conclude that cell uptake of HCV leads to viral RNA interaction with MAVS-dependent signaling actions of RIG-I. Thus, IFN induction in Kupffer cells could be different from its induction in plasmacytoid dendritic cells, of which signaling through TLR7/MyD88-dependent mechanisms serves to drive IFN expression by HCV. ${ }^{18}$

As a result of Kupffer cell IFN production, a high pretreatment ISG set point within hepatocytes likely mitigates the cellular response to IFN by driving a state of tolerance to ISG function, thus attentuating IFN actions and reducing the efficacy of therapy. We observed that the phase 1 HCV RNA decline within the first 24 hours after treatment with IFN- $a$ correlated with the treatment response at 12 weeks. Among patients with differential therapy responses, hepatic ISGs were expressed in specific patterns of uniform coexpression within responding cells, cell type or cell-specific expression, or focal/localized expression. Although the former pattern characterized our RVRs and EVRs, the other patterns were prevalent within the liver of the NRs and associated with a high hepatic ISG set point. Sarasin-Filipowicz et al noted that pretreatment ISG expression was not evident in PBMCs of NRs, ${ }^{13}$ and we show here the same pattern of response in PBMCs. Our results link the distinct patterns of hepatic ISG expression with therapy response outcome and suggest that an effective therapeutic response to IFN is facilitated by tissue-wide ISG induction whereas cell-specific and focal responses are limited in antiviral action. Thus, our results imply that ISG protein distribution patterns serve to direct the outcome of the phase 1 virologic response to IFN therapy. We examined the influences of the role of IL28B genotypes and treatment response in a separate study (data not shown) and found similar results to those first observed by Dill et $\mathrm{al}^{21}$ that high baseline ISG expression predicts the outcome of therapy more strongly than IL28B genotype for nonresponse. These results, together with the evidence of IFN- $\beta$ secretion by Kupffer cells, support the role of type 1 IFN in inducing the high basal ISG levels among nonresponders. However, it is important to evaluate the potential influences of type III IFN in these ISG activation pathways.

Bioinformatic analyses revealed that in the liver, the responding ISGs were linked through a central IRF-7 control node in RVRs and EVRs but not NRs, the latter of which exhibited ISG expression primarily through the STAT1/ISGF3 signaling network. This difference of ISG expression linked from an IRF-7 central node in responders but not NRs is an important distinction that may affect the outcome of IFN therapy. IRF-7 plays a major role in amplifying the IFN response through induction of IFN- $\alpha$ genes and by driving target ISG expression. ${ }^{3}$ This lack of IRF-7 signaling among NRs could be expected to attenuate the 
expression of specific ISGs that otherwise respond to IRF-7, thus rendering the cells "tolerant" to IFN therapy (see the following text). Unlike the hepatic response, ISG induction in PBMCs occurred simultaneously among RVRs, EVRs, and NRs. The separation of pretreatment ISG expression in the liver from PBMCs likely reflects the production of IFN by Kupffer cells and other endogenous myeloid cells of the liver induced through local exposure to HCV. This notion is supported by the assignment of STAT1 as the central node of ISG expression in pretreatment NR liver, indicating that initial IFN signaling but not amplification signaling was ongoing wherein the resulting cellular response imparts hepatic tolerance to therapeutic IFN- $\boldsymbol{a}$ application.

We modeled chronic $\mathrm{HCV}$ replication in the presence of constitutive low levels of IFN to validate the clinical observation of ISG tolerance in hepatocytes. Constitutive IFN- $a$ exposure in vitro generated a modest ISG set point level without an acute increase of ISG expression on re-treatment with IFN- $\boldsymbol{a}$. Furthermore, HCV RNA replication remained relatively constant with repeated IFN- $\alpha$ treatment, indicating that the cells became tolerant of IFN actions and/or that the HCV RNA replication process became tolerant to IFN. We show that IFN causes a reduction in HCV RNA levels that then stabilize during prolonged IFN exposure, likely reflecting tolerance to ISG expression and function. We propose a model of "innate immune tolerance" in NRs in which endogenous IFN drives basal ISG expression among hepatocytes. ISGs impart antiviral actions that when prolonged are cytotoxic $^{3,23}$ and, in the case of constitutive IFN exposure, ISG function must be tolerated for the cell to survive, thus forcing a state of tolerance to IFN (Figure 6). We do not precisely know why local IFN- $\beta$ production by Kupffer cells is more readily observed in NRs, but this could be due to an overall IFN amplification loop that enhances signaling through IFN priming. ${ }^{23}$

\section{Supplementary Material}

Refer to Web version on PubMed Central for supplementary material.

\section{Acknowledgments}

The authors thank Imad Nasser, MD, liver pathologist at Beth Israel Deaconess Medical Center, for the standardized scoring of the liver biopsy specimens and the nurses at the National Institutes of Health-sponsored General Clinical Research Center at Beth Israel Deaconess Medical Center and University of Texas Medical Branch at Galveston for their dedication in the care of the patients.

Funding

Supported by National Institutes of Health grants R01 DK068598-01 (to D.T.-Y.L.), AI028433, RR006555 (to A.S.P.), AI060389, DA024563, AI88778, P30DA015625 (to M.G.), AI40035 (to S.M.L.), M01-RR-01032, and M01-RR-01032 (General Clinical Research Center) and The Burroughs Wellcome Fund (to M.G.).

\section{Abbreviations used in this paper}

$\begin{array}{ll}\text { EVR } & \text { early virologic responder } \\ \text { GAPDH } & \text { glyceraldehyde-3-phosphate dehydrogenase } \\ \text { IFITM1 } & \text { interferon-induced transmembrane 1 } \\ \text { IFN } & \text { interferon } \\ \text { ISG } & \text { interferon-stimulated gene } \\ \text { NR } & \text { nonresponder } \\ \text { OAS } & \text { oligoadenylate synthetase }\end{array}$




$\begin{array}{ll}\text { PAMP } & \text { pathogen-associated molecular pattern } \\ \text { PBMC } & \text { peripheral blood mono-nuclear cell } \\ \text { RVR } & \text { rapid virologic responder } \\ \text { ShRNA } & \text { short hairpin RNA }\end{array}$

\section{References}

1. Ghany MG, Nelson DR, Strader DB, et al. An update on treatment of genotype 1 chronic hepatitis C virus infection: 2011 practice guideline by the American Association for the Study of Liver Diseases. Hepatology. 2011; 54:1433-1444. [PubMed: 21898493]

2. Schoggins JW, Wilson SJ, Panis M, et al. A diverse range of gene products are effectors of the type I interferon antiviral response. Nature. 2011; 472:481-485. [PubMed: 21478870]

3. Gale M Jr, Foy EM. Evasion of intracellular host defence by hepatitis C virus. Nature. 2005; 436:939-945. [PubMed: 16107833]

4. Edlich B, Ahlenstiel G, Zabaleta Azpiroz AZ, et al. Early changes in interferon signaling define natural killer cell response and refractoriness to interferon-based therapy of hepatitis $\mathrm{C}$ patients. Hepatology. 2012; 55:39-48. [PubMed: 21898483]

5. Sumpter R Jr, Wang C, Foy E, et al. Viral evolution and interferon resistance of hepatitis C virus RNA replication in a cell culture model. J Virol. 2004; 78:11591-11604. [PubMed: 15479800]

6. Neumann AU, Lam NP, Dahari H, et al. Hepatitis C viral dynamics in vivo and the antiviral efficacy of interferon-alpha therapy. Science. 1998; 282:103-107. [PubMed: 9756471]

7. Lam NP, Neumann AU, Gretch DR, et al. Dose-dependent acute clearance of hepatitis C genotype 1 virus with interferon alfa. Hepatology. 1997; 26:226-231. [PubMed: 9214474]

8. Lau DT, Fish PM, Sinha M, et al. Interferon regulatory factor-3 activation, hepatic interferonstimulated gene expression, and immune cell infiltration in hepatitis $\mathrm{C}$ virus patients. Hepatology. 2008; 47:799-809. [PubMed: 18203148]

9. Loo YM, Owen DM, Li K, et al. Viral and therapeutic control of IFN-beta promoter stimulator 1 during hepatitis C virus infection. Proc Natl Acad Sci U S A. 2006; 103:6001-6006. [PubMed: 16585524]

10. Fensterl V, Sen GC. Interferons and viral infections. Biofactors. 2009; 35:14-20. [PubMed: 19319841]

11. Thomas E, Gonzalez VD, Li Q, et al. HCV infection induces a unique hepatic innate immune response associated with robust production of type III interferons. Gastroenterology. 2012; 142:978-988. [PubMed: 22248663]

12. Wang C, Pflugheber J, Sumpter R Jr, et al. Alpha interferon induces distinct translational control programs to suppress hepatitis C virus RNA replication. J Virol. 2003; 77:3898-3912. [PubMed: 12634350]

13. Sarasin-Filipowicz M, Oakeley EJ, Duong FH, et al. Interferon signaling and treatment outcome in chronic hepatitis C. Proc Natl Acad Sci U S A. 2008; 105:7034-7039. [PubMed: 18467494]

14. Stienstra R, Saudale F, Duval C, et al. Kupffer cells promote hepatic steatosis via interleukin-1beta-dependent suppression of peroxisome proliferator-activated receptor alpha activity. Hepatology. 2010; 51:511-522. [PubMed: 20054868]

15. Pflugheber J, Fredericksen B, Sumpter R Jr, et al. Regulation of PKR and IRF-1 during hepatitis C virus RNA replication. Proc Natl Acad Sci U S A. 2002; 99:4650-4655. [PubMed: 11904369]

16. Sarkar SN, Sen GC. Novel functions of proteins encoded by viral stress-inducible genes. Pharmacol Ther. 2004; 103:245-259. [PubMed: 15464592]

17. Sumpter R Jr, Loo YM, Foy E, et al. Regulating intracellular antiviral defense and permissiveness to hepatitis C virus RNA replication through a cellular RNA helicase, RIG-I. J Virol. 2005; 79:2689-2699. [PubMed: 15708988] 
18. Takahashi K, Asabe S, Wieland S, et al. Plasmacytoid dendritic cells sense hepatitis C virusinfected cells, produce interferon, and inhibit infection. Proc Natl Acad Sci U S A. 2010; 107:7431-7436. [PubMed: 20231459]

19. Chen L, Borozan I, Feld J, et al. Hepatic gene expression discriminates responders and nonresponders in treatment of chronic hepatitis C viral infection. Gastroenterology. 2005; 128:1437-1444. [PubMed: 15887125]

20. Feld JJ, Nanda S, Huang Y, et al. Hepatic gene expression during treatment with peginterferon and ribavirin: identifying molecular pathways for treatment response. Hepatology. 2007; 46:15481563. [PubMed: 17929300]

21. Dill MT, Duong FH, Vogt JE, et al. Interferon-induced gene expression is a stronger predictor of treatment response than IL28B genotype in patients with hepatitis C. Gastroenterology. 2011; 140:1021-1031. [PubMed: 21111740]

22. Chen L, Borozan I, Sun J, et al. Cell-type specific gene expression signature in liver underlies response to interferon therapy in chronic hepatitis C infection. Gastroenterology. 2010; 138:11231133. [PubMed: 19900446]

23. Loo YM, Gale M Jr. Viral regulation and evasion of the host response. Curr Top Microbiol Immunol. 2007; 316:295-313. [PubMed: 17969453] 
A

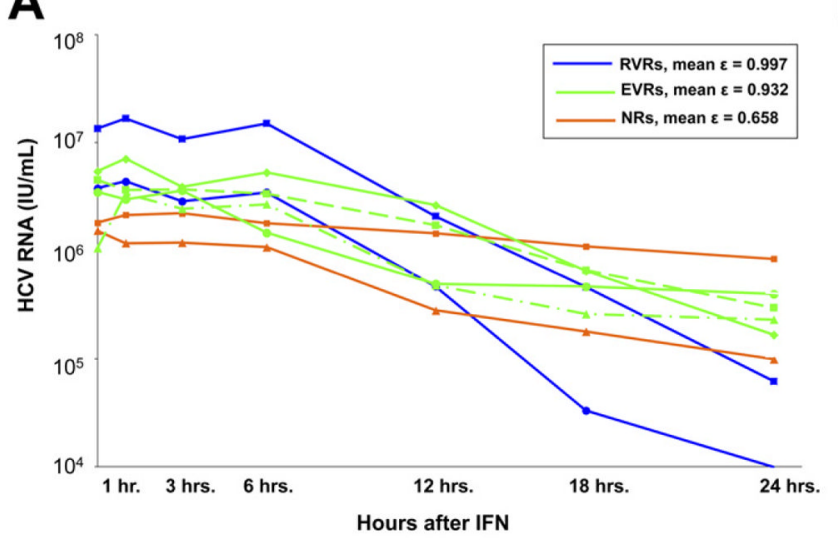

C

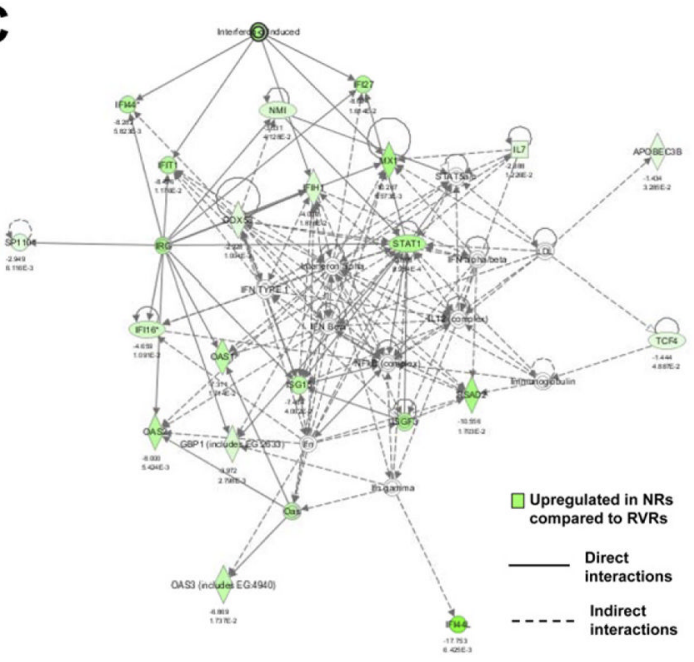

B

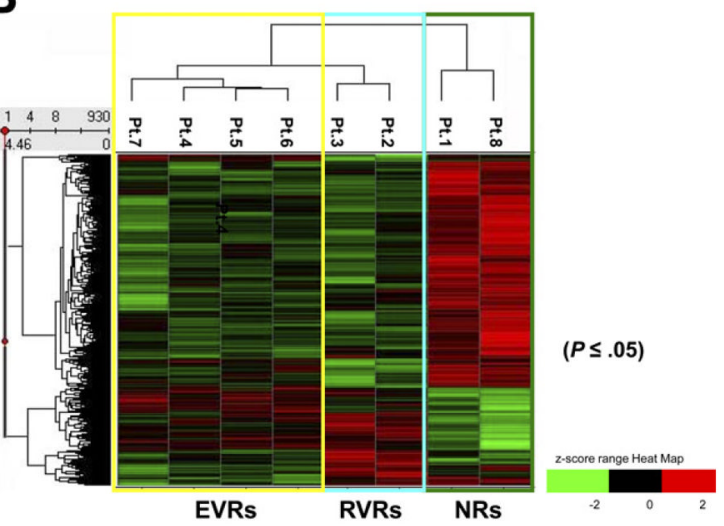

D

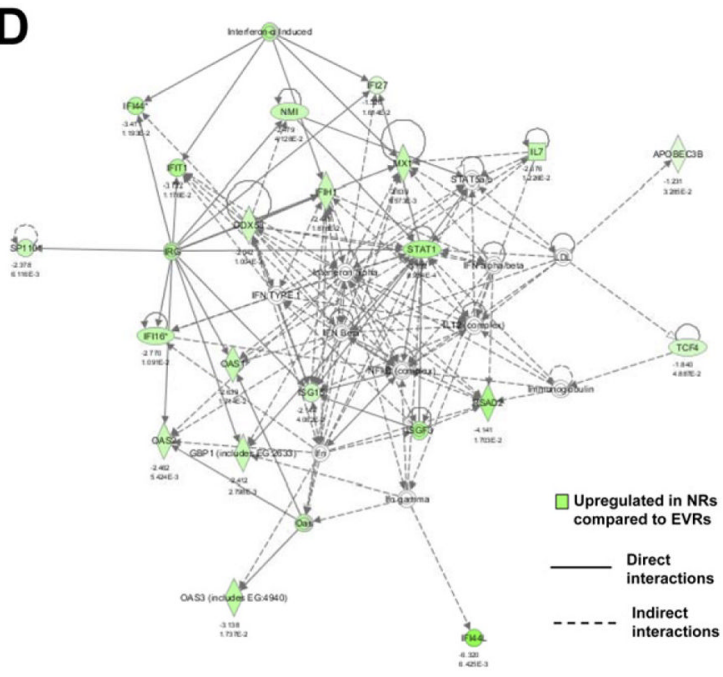

Figure 1.

(A) Phase 1 HCV RNA kinetic after a single dose of IFN- $\alpha$-2a (10 MU subcutaneously). $(B-D)$ Induction of differential hepatic genes and ISGs before administration of IFN- $a$. $(B)$ A heat map of RVRs, EVRs, and NRs shows distinct gene clusters. A total of $68 \%$ of the significant genes were up-regulated in NRs. Ingenuity Pathways Analysis of ISG response comparing $(C)$ RVRs with NRs and $(D)$ EVRs with NRs. 
A

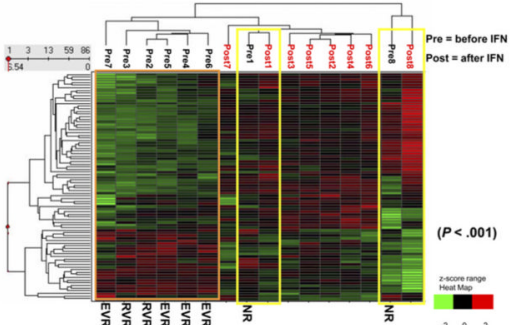

B

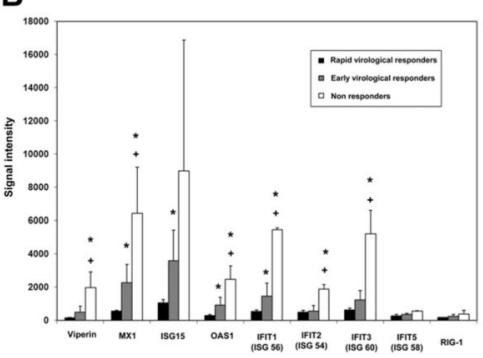

C
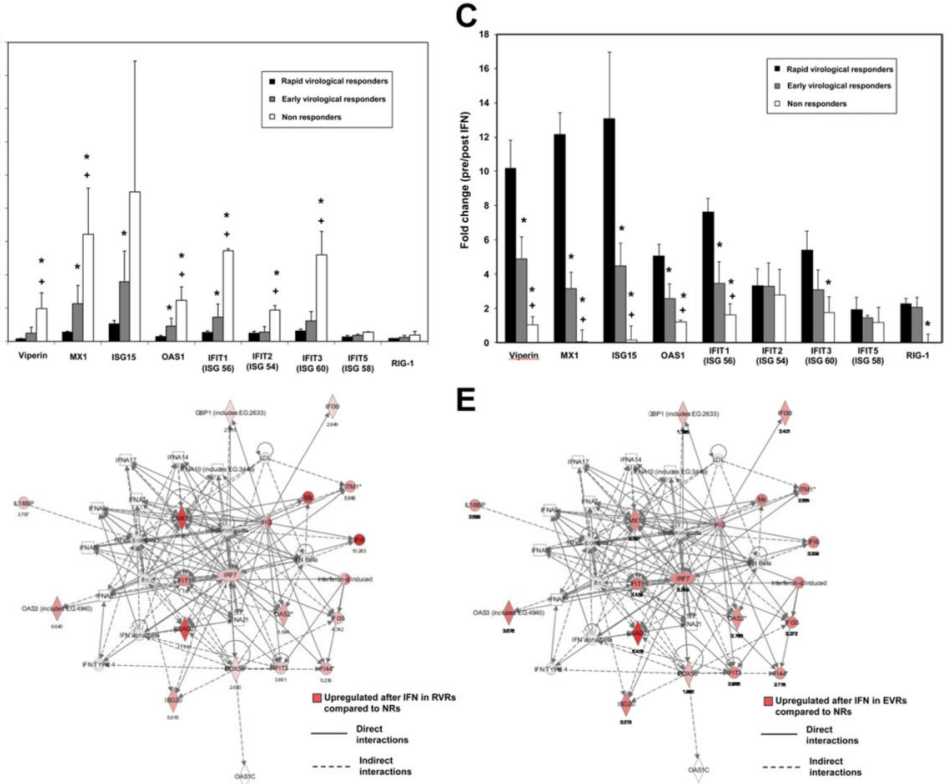

E

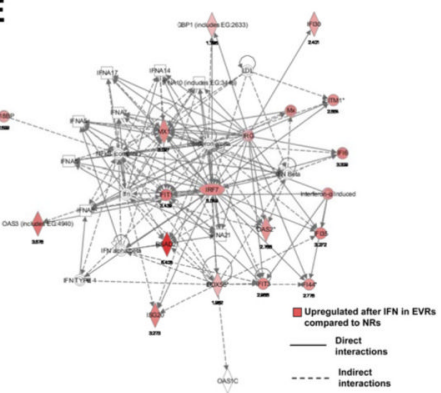

Figure 2.

Differential hepatic ISG induction after administration of IFN- $a$. $(A)$ Heat map. $(B)$

Selective ISG signal intensity before administration of IFN- $\alpha$ (mean \pm SD). ${ }^{*} P<.05$ (RVR vs NR, or RVR vs EVR); ${ }^{+} P<.05$ (EVR vs NR). ( $C$ ) Fold changes (mean $\pm \mathrm{SD}$ ) in hepatic ISG after administration of IFN- $a{ }^{*} P<.05$ (RVR vs NR, or RVR vs EVR); ${ }^{+} P<.05$ (EVR vs NR). Ingenuity Pathways Analysis of up-regulated genes after administration of IFN- $\alpha$ in $(D)$ RVRs compared with NRs and $(E)$ EVRs compared with NRs. 


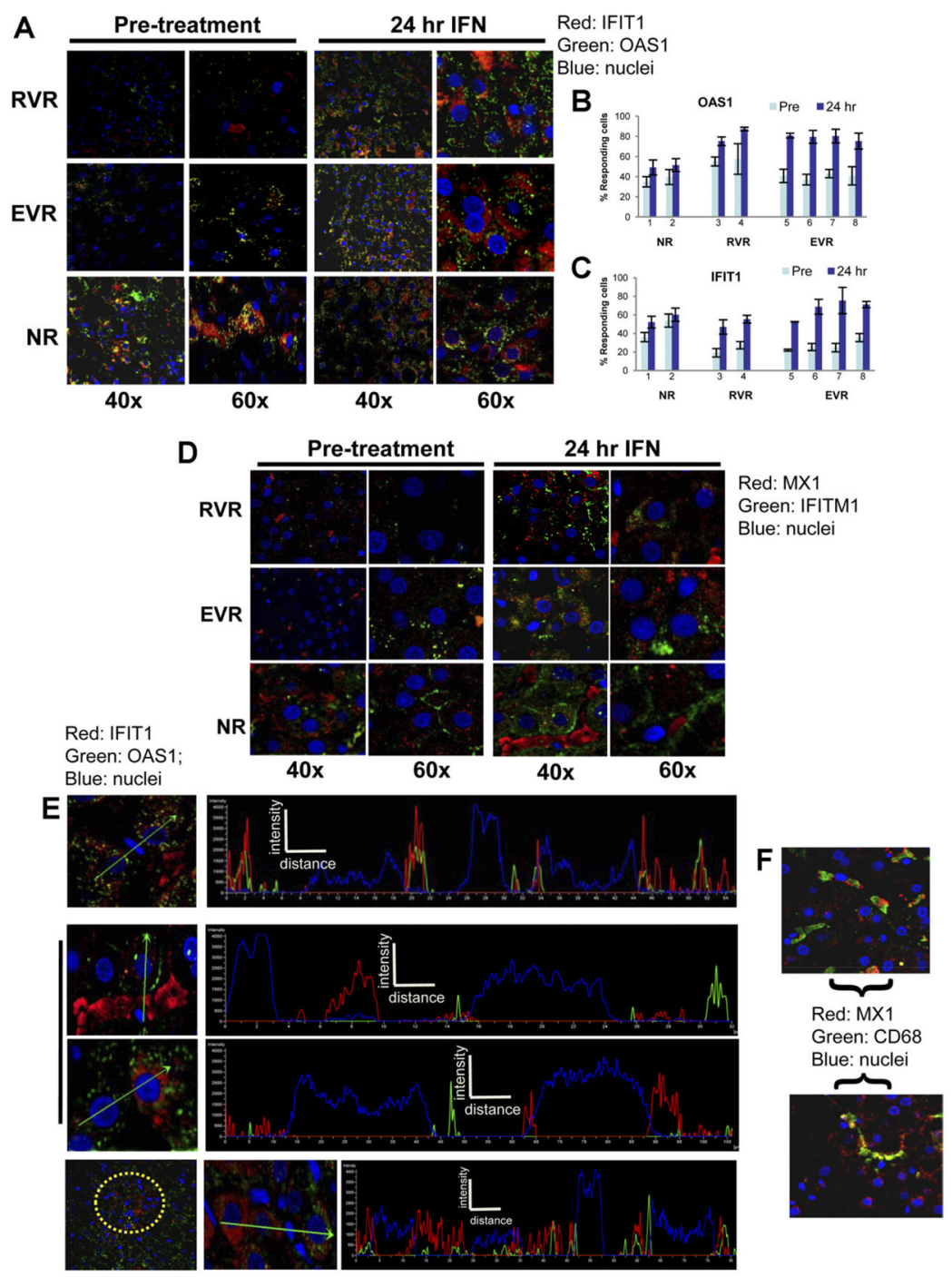

Figure 3.

Hepatic ISG expression distribution patterns in pretreatment and IFN-treated patients with $\mathrm{HCV}$ ( bar $=10 \mu \mathrm{mol} / \mathrm{L})$. (A) Merged color confocal images of $0.2-\mu \mathrm{m}$ liver sections showing IFIT1 (red), OAS1 (green), and nuclei (blue). ( $B$ and $C$ ) Percent responding cells expressing $(B)$ OAS or $(C)$ IFIT1 in liver sections from each patient group (mean $\pm \mathrm{SD}$ ). Numbers were determined by counting stained cells within 10 different 40x magnification fields from the patients in each group. $(D)$ ISG protein distribution in $0.2-\mu \mathrm{m}$ liver sections showing merged confocal color images of immunostained tissue: MX1 (red), IFITM1 (green), and nuclei (blue). ( $E$ and $F$ ) Merged color confocal images showing different patterns of IFIT1 (red) and OAS1 (green) distribution with nuclei (blue) in IFN-treated liver. (E) Fluorescence signal intensity within the tissue distance denoted by vector (arrow) or focal region (circle) in the far left panels is shown in histogram form in the accompanying right panels. The top panels show confluent and overlapping distribution from an RVR, and the middle panels show cell-specific distribution in an NR featuring differential ISG expression in hepatocytes and neighboring Kupffer cells (upper middle panel) or among adjacent hepatocytes (lower middle panel). The bottom panels show focal distribution of ISG expression within responding hepatocyte clusters among background of nonresponding cells in an NR. Histograms correspond to the signal intensity from specific costaining of 
nuclei (blue), IFIT1 (red), and OAS1 (green). $(F)$ Merged micrograph of a pretreatment NR patient liver showing nuclei (blue), MX1 (red), and CD68 (identifying Kupffer cells; green). 

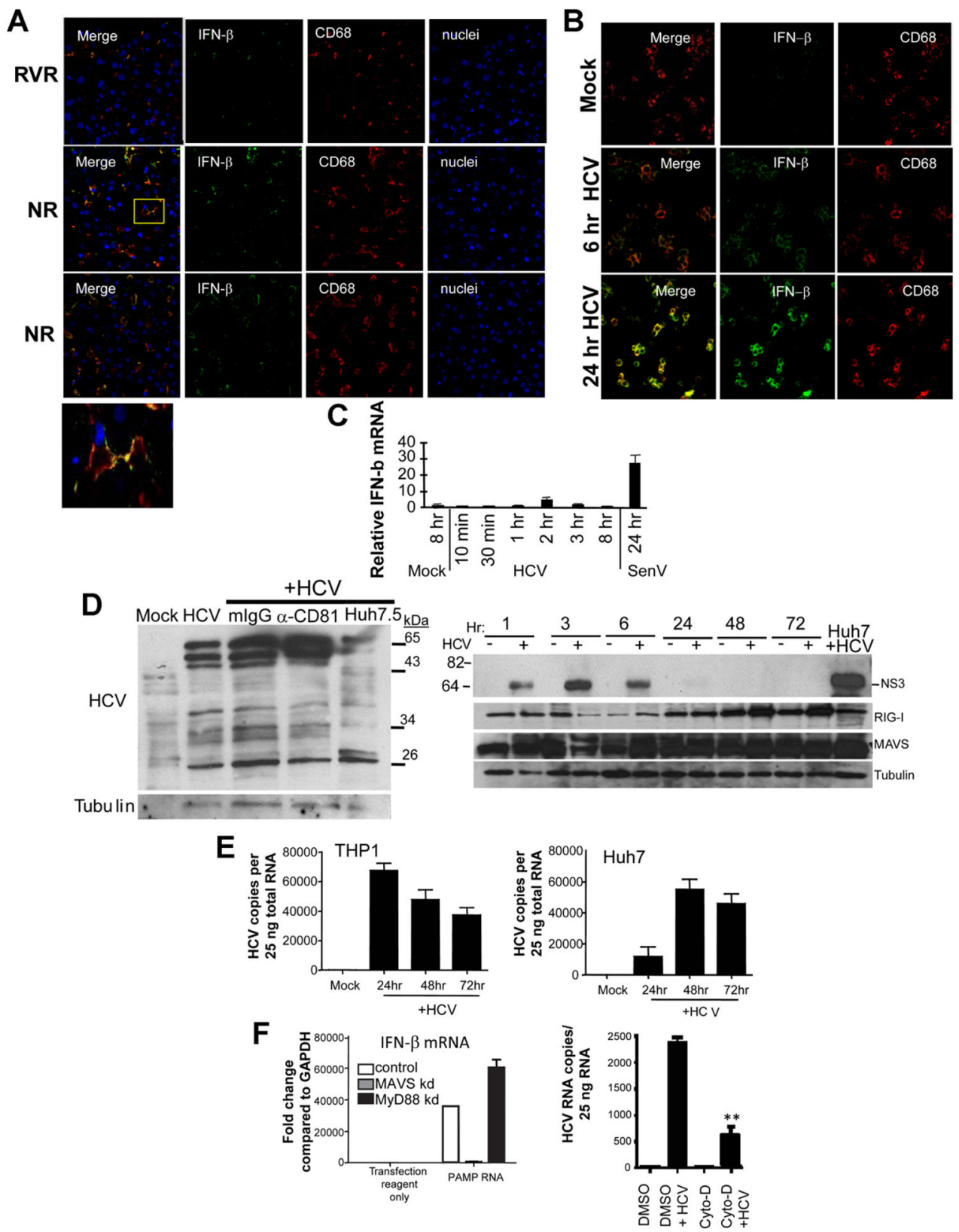

Figure 4.

IFN- $\beta$ and CD68 expression. ( $A$ ) Protein distribution in pretreatment HCV patient liver. Liver sections were stained with anti-IFN- $\beta$ (green), anti-CD68 (red), and nuclei (blue). Panels show $0.3-\mu \mathrm{m}$ optical sections of $40 \times$ merged confocal color images from an RVR (upper panel) and 2 different NRs (lower panels). The far right panel shows a $60 \times$ close-up of the area bounded by the yellow box in the NR panel. $(B)$ Distribution of IFN- $\beta$ and CD68 in immunostained THP1 cells. Cells were either mock treated or cultured with HCV (JFH1) for 6 or 24 hours as indicated. HCV exposure of cells was based on a theoretical multiplicity of infection (MOI) of 0.1 as determined by virus titration in Huh7 cells. Similar results were obtained from THP1 cells exposed to HCV at an MOI of 0.3 (data not shown). ( $C$ ) Fold change (mean+/- SD) of IFN- $\beta$ mRNA expression in mocked or HCV-exposed THP1 cells. (D) HCV protein expression in cultured cells. (Left panel) HCV protein expression in THP1 
or Huh7.5 cells (lane 5) 36 hours after purified culture media mock or HCV (MOI of 0.1) exposure. $\mathrm{mIg}$ and anti-CD81 denote cells pretreated with control immunoglobulin or antiCD81 blocking antibody before exposure to virus. (Right panel) THP1 cells were exposed to media alone or HCV (MOI of 0.1) for the time indicated in hours. Panels show HCV NS3, RIG-I, MAVS, and tubulin expression. The far right lane shows protein expression in HCVinfected Huh7 cells. (E) (Left paneI) HCV RNA levels in THP1 cells after mock or HCV exposure shown in hours. No viral RNA was detectable by 96 hours after HCV exposure (data not shown). HCV copy number (mean \pm SD). (Right panel) HCV RNA levels in Huh7 cells shown in hours after HCV exposure. HCV copy number (mean $\pm \mathrm{SD}$ ). ( F) (Left panel) IFN- $\beta$ mRNA levels in THP1 cells harboring nontargeting shRNA (control) or shRNA to knock down (kd) the expression of MAVS or MyD88. Cells were treated with transfection reagent alone (left panel) or transfected with the 100-nucleotide poly-U/UC PAMP RNA motif encoded within the HCV genome RNA (PAMP RNA; right panel). Fold change (mean $\pm \mathrm{SD}$ ). Level of mRNA expression knockdown was compared with nontargeting shRNA control and was $>99 \%$ for MAVS shRNA and 90\% for MyD88 shRNA as determined by reverse-transcription quantitative polymerase chain reaction analysis of specific mRNA present. (Right panel) HCV RNA levels in HCV-treated THP1 cells that were pretreated with dimethyl sulfoxide or cytochalasin D alone for 30 minutes before HCV exposure. HCV RNA level was determined by reverse-transcription quantitative polymerase chain reaction and compared with GAPDH. ${ }^{* *} P=.03$. 

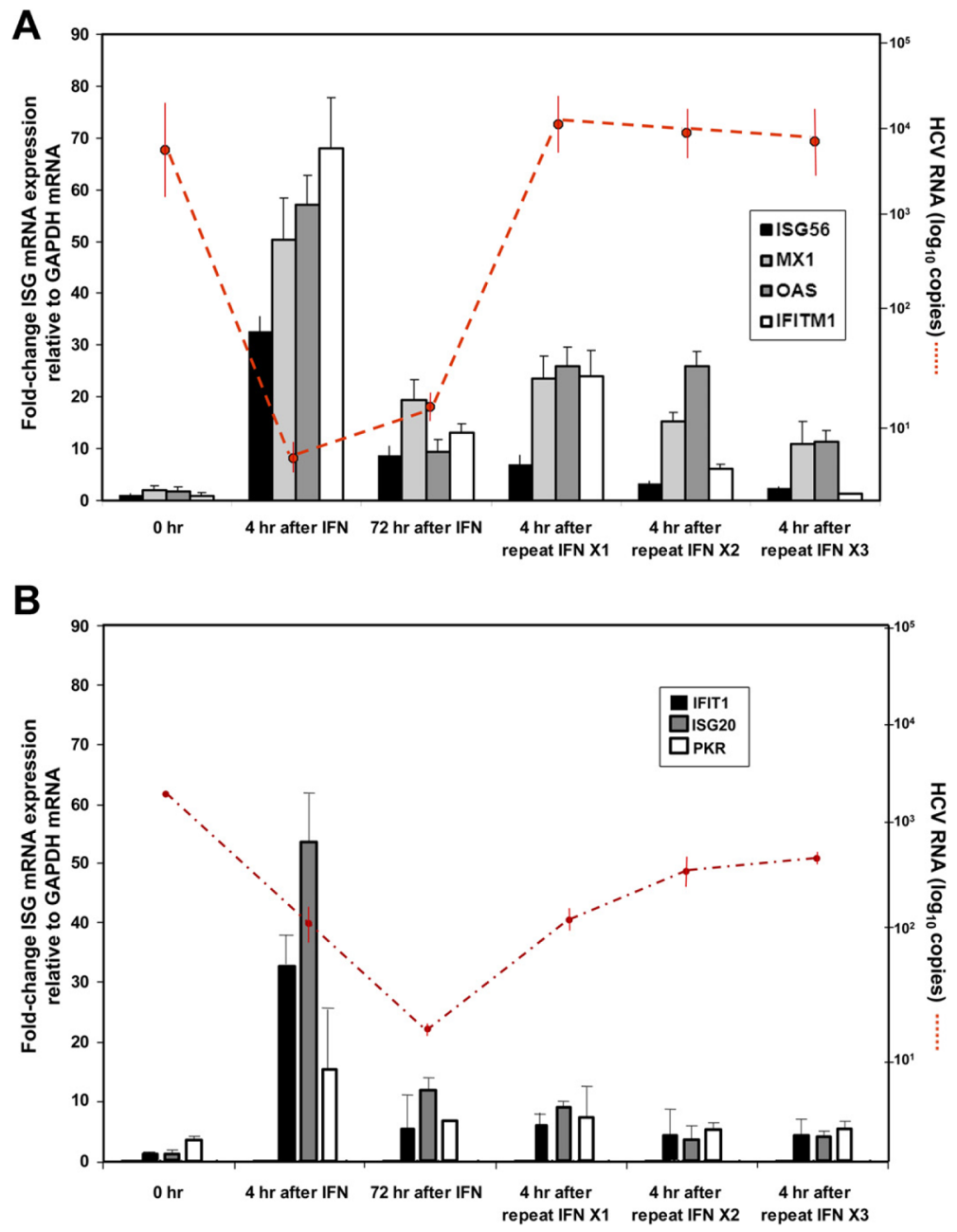

Figure 5.

In vitro model of acute and constitutive IFN response. $(A)$ HCV RNA levels as compared with GAPDH and ISG (IFIT1, MX1, OAS1, and IFITM1) expression during repeated treatment with IFN- $\alpha$ in Huh7-K2040 cells (mean \pm SD). (B) HCV RNA levels as compared with GAPDH and ISG (IFIT1, ISG20, and PKR) expression during repeated treatment with IFN- $\alpha$ in Huh7-L2198S cells (mean \pm SD). 


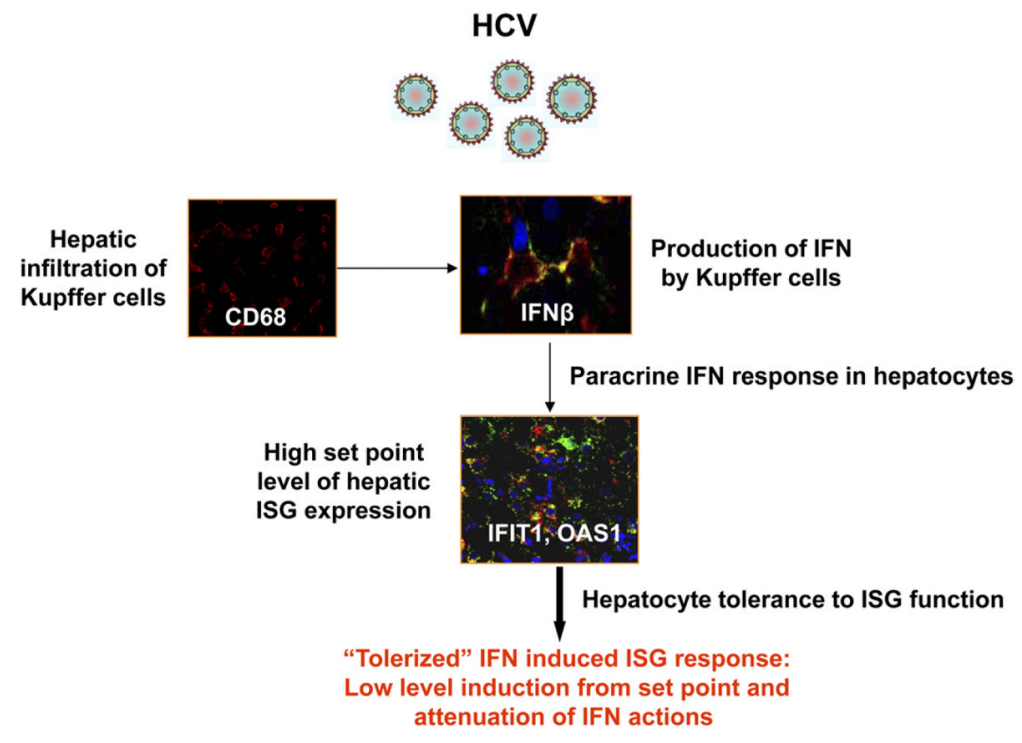

Figure 6.

Innate immune tolerance model in the treatment of chronic hepatitis C. Endogenous IFN- $\beta$ produced by myeloid cells drives basal ISG expression among hepatocytes via paracrine signaling. ISGs impart antiviral actions through multiple processes, which are often cytotoxic with prolonged exposure. ISGs, therefore, must be tolerated for the cell to survive. As the cell becomes tolerized to the actions of IFN, the efficacy of IFN therapy is reduced. 